\title{
The application of imaging technologies in the detection of trace evidence in forensic medical investigation
}

\author{
Jeannie Cocks $^{\mathrm{a}}{ }^{*},{ }$, Lorraine du Toit-Prinsloo $^{\mathrm{a}}$, Francois Steffens $^{\mathrm{b}}{ }$, Gert Saayman ${ }^{\mathrm{a}}$ \\ ${ }^{a}$ Department of Forensic Medicine, University of Pretoria, Private Bag X323, Arcadia 0007, South \\ Africa \\ ${ }^{\mathrm{b}}$ Department of Statistics, University of Pretoria, Private Bag X20, Hatfield 0028, South Africa
}

South Africa.

* Corresponding author at: PO Box 1381, Bedfordview 2008, South Africa. Tel.: +27 73171 2031. Email address: jeaniusofthelamp@hotmail.com (J. Cocks).

\begin{abstract}
In a country notorious for violent crime, it seems that South African medico-legal laboratories make minimal application of technology in the death investigation process and little attention is given to trace evidence. Non-destructive, non-invasive, portable and cost-effective tools are required. This study was conducted at the Pretoria Medico-Legal Laboratory. The surface area of the bodies and clothing of victims of fatal interpersonal violence were examined using a torch, magnifying lamp, portable digital microscope and alternate light source to gauge their potential for trace evidence detection. Most studies apply these and similar tools to inert surfaces, with few focusing on their application to human skin. There was a statistically significant difference in the detection of many of the evidence types between the naked-eye observation of the pathologists and the technologies. The different imaging technologies were compared as to their cost, evidence detection ability and ease of use. The most common evidence types discovered on the bodies and clothing of victims of fatal interpersonal violence, as well as the propensity of each tool to detect these, was evaluated in order to devise the best option for incorporation into the Pretoria Medico-Legal Laboratory routine. The digital microscope performed best overall followed by the magnifying lamp, torch and the Polilight@ ${ }^{\circledR}$. This study aimed to justify the investment of more time, effort and funding into trace evidence recovery in the South African mortuary environment.
\end{abstract}

\section{Keywords}

Trace evidence; imaging technology; Alternate Light Source; evidence detection; forensic medical investigation

\section{Introduction}

Locard's Exchange Principle: "Every contact leaves a trace" encapsulates the fact that evidence found at a crime scene can create links between the perpetrator and the victim.[1] There is virtually no limit to the traces that could be found on a victim's body, albeit each with varying degrees of uniqueness and subsequent usefulness to the investigation. This evidence can prove that a suspect came in contact with the victim around the time of the 
crime, and can subsequently serve to incriminate or exonerate the individual.[2] In fatal cases, the body should be treated with the same care, diligence and vigilance as the physical crime scene; and trace evidence should be meticulously sought for in the same manner. In homicidal cases and pedestrian vehicle accidents (PVA) there is potential contact between the victim and the offender and/or weapon. Homicide accounts for over 500000 deaths per year worldwide and 270000 pedestrians lose their lives on the roads each year.[3-4]

Burton (2007)[5] considers the initial external examination of victims' bodies to be neglected, poorly documented and not routinely thoroughly inspected for trace evidence in mortuaries.[5] The medico-legal investigation of death is in need of ancillary testing technologies which deliver reproducible, reliable results and are non-destructive.[2] Factors such as cost, ease of use and portability need to be considered when choosing imaging technologies for trace evidence recovery. Pretoria is the capital city of South Africa with an estimated population of 2141717 (2007).[6] This study was conducted at the Pretoria Medico-Legal Laboratory (PMLL), which admits the majority of cases from Pretoria. A torch, magnifying lamp, portable digital microscope and alternate light source were tested to gauge their potential for trace evidence detection on the bodies of victims of fatal interpersonal violence.

\section{Material and methods}

\section{Setting}

This was a prospective study conducted over a 6 month period at the PMLL. The study proposal was approved by the University of Pretoria's Faculty of Health Sciences' Research Ethics Committee, the MSc Committee and the relevant authorities at the PMLL.

\section{Case Selection}

Any case where it was considered by the researcher and/or attending pathologist that there may have been some sort of violent interaction resulting in a possible transfer of physical evidence was included in the study. This included victims of homicidal blunt-force and sharpforce trauma, manual and/or ligature strangulation and a number of pedestrian-vehicle accidents (PVA).

Cases where perpetrator-victim contact was expected to be minimal (such as gunshotwounds and vehicle occupants in road-traffic accidents), as well as decedents who were hospitalized, were excluded.

\section{Technologies}

Four technologies were tested in this study. The first was a torch, the LED Lenser® M7 (R500.00-R800.00). The torch makes use of "High End Power LED"s and is $137 \mathrm{~mm}$ long and weighs $193 \mathrm{~g}$. It produces 220 Lumens and operates on 4 AAA batteries. It has a burning life of 11 hours and a beam range of $255 \mathrm{~m}$.

A magnifying lamp was used secondly (model number MLPF8066-1BHC) (R400.00$\mathrm{R} 1000.00$ ). It has a $125 \mathrm{~mm}$ diameter, 8,3 dioptre glass lens allowing for $\times 3$ magnification. The arm length is $410 \mathrm{~mm}$ and the entire unit clamps onto a desktop. It operates from a $220 \mathrm{~V}$ mains supply and has a fluorescent ring light surrounding the lens. 
Thirdly, the Veho VMS-004 USB Microscope (digital microscope) was used (R300.00$\mathrm{R} 2000.00$ ). It has dimensions of $125 \mathrm{~mm} \times 33 \mathrm{~m}$, with a $1.3 \mathrm{Mega}$ Pixel image sensor and still- and video-capture capabilities. It has a manual focus range from $10 \mathrm{~mm}$ to $500 \mathrm{~mm}$ and a magnification ratio of 20x and 400x. Illumination is provided by an 8 LED light source which can be adjusted by a control wheel. The microscope is powered via the USB port in a computer. Microcapture software is included which allows approximate measurements to be calculated on the images.

A forensic light source (Alternative Light Source) was also procured on indefinite loan from the South African Police Service (SAPS). The unit used was a Polilight@ PL500 from Rofin Australia (Pty) LTD (R38 000.00-R450 000.00). It is a 500 Watt high-intensity Xenon light source with dimensions of $33 \times 15 \times 37 \mathrm{~cm}$ and weighing $9.9 \mathrm{~kg}$. It uses a $2 \mathrm{~m}$ long flexible liquid light guide and 12 selectable and tuneable filters to generate light of varying wavelengths. It uses a standard power supply ranging from $90-260$ volts and $50-60 \mathrm{~Hz}$. Four pairs of coloured goggles accompany the unit for the user's protection. Coloured camera filters are also provided to allow documentation of findings.

\section{Methodology}

The body of each victim included in the study was viewed by the attending pathologist in the medico-legal mortuary and then subsequently undressed by the attending prosector. The bodies were not washed or cleaned in any way and the body was then moved to an adjacent room for examination purposes.

The clothing and body were examined with the technologies in the following order: first using the torch, then the magnifying light, the digital microscope and lastly the Polilight ${ }^{\circledR}$. The rationale for the order of use of the technologies was intended to go from perceived weakest - and therefore least likely to detect evidence - to strongest, to try and eliminate the bias of seeking out already-found evidence. The digital microscope was used at $20 x$ magnification and was connected to an HP ProBook 4515s laptop using Windows $\AA 7$ Home Basic. The Polilight ${ }^{\circledR}$ was set at $450 \mathrm{~nm}$ at full power (P8) in conjunction with orange filter goggles.

The clothing was laid out on a workbench in the room for inspection by the forensic scientist. One scientist did all the examinations in order to eliminate inter-observer discrepancies. Just the outer layers of clothing - where contact was expected to occur - were examined. The examination of the clothing took an average of 30 minutes.

The bodies were subsequently examined using the technologies in the same order as for the clothing. The anterior aspect of the body was examined first (with all 4 technologies) where after the body was turned over in order to examine the posterior aspect of the body. It took approximately 60 minutes to examine the entire surface area of the body with all 4 technologies. Evidence that was discovered was noted. Evidence was divided into the following categories: fibres/hairs, fluid (this did not include what appeared to be condensation from refrigeration), geological samples (gravel, sand, dirt, etc.), botanical samples (grass, leaves, seeds, etc.), paint, glass, impression marks (areas of constriction or pressure that may highlight areas worth investigating for touch DNA, for example in cases where a victim was throttled), entomological samples (insects, maggots, etc.) fingerprints, tattoos, plastic and paper. Any other traces, such as smears, flecks, powders, mould, soot/ash etc., were grouped into the category of 'other evidence'. The traces were grouped 
in this manner in order to simplify the results and because they could not be definitely and uniquely identified without further testing. The collection of samples and further confirmatory testing was beyond the scope of the study; therefore evidence found could only be given assumptive descriptions and may not have been their true identities. Certain crimes or circumstances of death may show correlation with certain evidence types, for example, body fluids and rape-homicides. This categorization allows one to identify particular technologies which would be of the most use for a particular case type or external cause of death.

The attending pathologist was informed of the evidence discovered through the examinations and it was left to their discretion whether or not to collect samples. Interesting findings or representative examples were photographed. Evidence noted in the subsequent pathologists' reports was taken as naked-eye observations for comparative purposes.

The different imaging technologies were compared as to their cost, evidence detection ability and ease of use.

\section{Statistical Analysis}

Assistance with statistical analysis was sought from the Statistics Department at the University of Pretoria. The IBM SPSS Statistics Version $21 \AA$ program was used for the statistical analysis. The approach was a pairwise comparison of technologies for each type of evidence.

In order to compare the efficacy of two devices to find evidence, the McNemar Test was used because the same bodies were searched using the different technologies, making the observations paired observations. In comparing Technology $A$ to Technology $B$, the number of times evidence was found by means of Technology $A$ and not by $B$ is compared to the number of times evidence was found by means of Technology $B$ but not by means of Technology A. The evidence found by both or by neither plays no role in this comparison. McNemar Tests could not be performed in the cases where one or both technologies being compared failed to find anything in that evidence category. A p-value of less than 0.05 was taken as indication of a significant difference.

\section{Results}

\section{Study Population}

The final case total amounted to 55 cases. Table I summarizes all the results pertaining to each case including external cause of death, demographic details and type of traces found.

\section{External Cause/Circumstance of Death}

The 55 selected cases comprised of 21 (38.2\%) victims of sharp force assault, 14 (25.5\%) victims of blunt force assault, $8(14.5 \%)$ PVAs, $6(10.9 \%)$ victims of multiple forms of trauma, $3(5.5 \%)$ victims of manual strangulation, $1(1.8 \%)$ victim of ligature strangulation, $1(1.8 \%)$ victim who was gagged, and $1(1.8 \%)$ victim who was a cyclist hit by a car. Seven $(12.7 \%)$ cases were suspected to include an element of rape or sexual assault. 


\section{Demographics}

The majority of victims were male (78.2\%). Forty-six (83.6\%) victims were Black Africans, 7 (12.7\%) victims were White and $2(3.6 \%)$ victims were Coloured. It is recognized that the terms 'Black', 'White' and 'Coloured' have no scientific basis and are merely social classifications of various population groups.

Table I. Demographic information and evidence found per case.

\begin{tabular}{|c|c|c|c|c|c|c|c|c|c|c|c|c|c|c|}
\hline & Demogr & phic & & & & & & vide & ce F & und & & & & \\
\hline 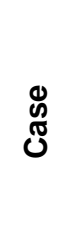 & 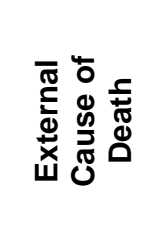 & $\stackrel{\times}{\infty}$ & 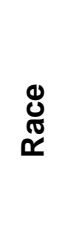 & 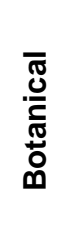 & 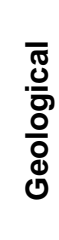 & 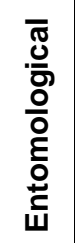 & $\begin{array}{l}\text { D } \\
\frac{\mathbb{O}}{\sigma} \\
\end{array}$ & $\frac{\overrightarrow{\frac{1}{\sigma}}}{a}$ & 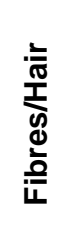 & $\frac{\text { 을 }}{\frac{3}{4}}$ & 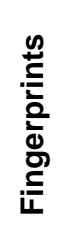 & 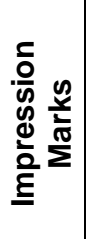 & 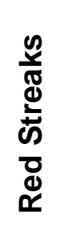 & 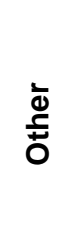 \\
\hline 1 & Multiple & M & B & $\checkmark$ & $\checkmark$ & & & $\checkmark$ & & & & & & \\
\hline 2 & PVA & $\mathrm{F}$ & $B$ & & $\checkmark$ & & & & & $\checkmark$ & & & & \\
\hline 3 & Sharp & $M$ & $\mathrm{~B}$ & $\checkmark$ & $\checkmark$ & & & & & & & & & $\checkmark$ \\
\hline 4 & Multiple & $M$ & $W$ & & & & & & $\checkmark$ & $\checkmark$ & & & & $\checkmark$ \\
\hline 5 & PVA & $M$ & $B$ & $\checkmark$ & 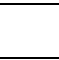 & & & $\checkmark$ & $\checkmark$ & $\checkmark$ & & & & $\checkmark$ \\
\hline 6 & Multiple & $\mathrm{F}$ & $B$ & $\checkmark$ & $\checkmark$ & & & $\checkmark$ & $\checkmark$ & $\checkmark$ & & & & $\checkmark$ \\
\hline 7 & Blunt & $M$ & B & $\checkmark$ & $\checkmark$ & & & $\checkmark$ & $\checkmark$ & $\checkmark$ & & & & $\checkmark$ \\
\hline 8 & Blunt & $M$ & $B$ & & $\checkmark$ & & & & $\checkmark$ & $\checkmark$ & & & & $\checkmark$ \\
\hline 9 & Sharp & $M$ & W & $\checkmark$ & & & & $\checkmark$ & $\checkmark$ & $\checkmark$ & & & & \\
\hline 10 & Manual & $\mathrm{F}$ & $B$ & $\checkmark$ & & & & & $\checkmark$ & $\checkmark$ & & & & $\checkmark$ \\
\hline 11 & Manual & $\mathrm{F}$ & $B$ & $\checkmark$ & $\checkmark$ & & & & $\checkmark$ & $\checkmark$ & & & & \\
\hline 12 & Multiple & $M$ & $B$ & $\checkmark$ & & & & & $\checkmark$ & $\checkmark$ & & & & $\checkmark$ \\
\hline 13 & Sharp & $M$ & $\mathrm{C}$ & & & & & & & & & & & \\
\hline 14 & PVA & $M$ & $w$ & & $\checkmark$ & & $\checkmark$ & & $\checkmark$ & & & & & \\
\hline 15 & PVA & $M$ & $\mathrm{~B}$ & & & & $\checkmark$ & & $\checkmark$ & & & & & \\
\hline 16 & Sharp & $M$ & $B$ & & $\checkmark$ & & & & $\checkmark$ & & & & & \\
\hline 17 & Sharp & $M$ & $B$ & & & & & & & $\checkmark$ & & & & \\
\hline 18 & Multiple & $\mathrm{F}$ & W & & & & & & $\checkmark$ & & & & & \\
\hline 19 & Sharp & $\mathrm{F}$ & $w$ & & & & & & $\checkmark$ & & & & & \\
\hline 20 & Sharp & $M$ & $B$ & $\checkmark$ & $\checkmark$ & & & & & $\checkmark$ & & & & \\
\hline 21 & Blunt & $M$ & $B$ & & $\checkmark$ & & & & $\checkmark$ & & & & & \\
\hline 22 & Sharp & $M$ & $B$ & $\checkmark$ & $\checkmark$ & & & & $\checkmark$ & & & & & \\
\hline 23 & Blunt & $M$ & $B$ & $\checkmark$ & & & & & & $\checkmark$ & & $\checkmark$ & & $\checkmark$ \\
\hline 24 & Blunt & $M$ & $B$ & & $\checkmark$ & & & & & $\checkmark$ & & & & \\
\hline 25 & Blunt & $M$ & $B$ & $\checkmark$ & & & & & $\checkmark$ & & & & & \\
\hline 26 & Sharp & $M$ & $B$ & & $\checkmark$ & & & & $\checkmark$ & $\checkmark$ & & & & $\checkmark$ \\
\hline 27 & Sharp & $M$ & $B$ & $\checkmark$ & & & & & & $\checkmark$ & & & & \\
\hline 28 & Blunt & $M$ & $B$ & & & & & & & $\checkmark$ & & & & \\
\hline 29 & Sharp & $M$ & $B$ & $\checkmark$ & & & & & & & & & & \\
\hline 30 & Sharp & $M$ & $B$ & & & & & & & $\checkmark$ & & & & \\
\hline 31 & Blunt & $M$ & $B$ & & $\checkmark$ & & & & $\checkmark$ & & & & & $\checkmark$ \\
\hline
\end{tabular}




\begin{tabular}{|l|c|c|c|c|c|c|c|c|c|c|c|c|c|c|}
\hline 32 & Blunt & M & B & $\checkmark$ & $\checkmark$ & & $\checkmark$ & $\checkmark$ & $\checkmark$ & $\checkmark$ & & & & $\checkmark$ \\
\hline 33 & Multiple & F & B & $\checkmark$ & $\checkmark$ & & & & $\checkmark$ & $\checkmark$ & & & & \\
\hline 34 & Sharp & M & B & & & & & & $\checkmark$ & $\checkmark$ & & & & \\
\hline 35 & Sharp & M & B & & $\checkmark$ & & $\checkmark$ & & $\checkmark$ & & & & & \\
\hline $\mathbf{3 6}$ & Blunt & M & B & & $\checkmark$ & & & & & & & & & \\
\hline $\mathbf{3 7}$ & Sharp & F & B & & $\checkmark$ & & & $\checkmark$ & $\checkmark$ & $\checkmark$ & & & & $\checkmark$ \\
\hline $\mathbf{3 8}$ & Gagging & F & W & & & & & & & & & & & $\checkmark$ \\
\hline $\mathbf{3 9}$ & Blunt & M & B & $\checkmark$ & $\checkmark$ & & & & $\checkmark$ & & & & & $\checkmark$ \\
\hline $\mathbf{4 0}$ & PVA & M & B & & $\checkmark$ & & & & $\checkmark$ & $\checkmark$ & & & & \\
\hline $\mathbf{4 1}$ & Sharp & M & B & & & & & & $\checkmark$ & & & & & \\
\hline $\mathbf{4 2}$ & Sharp & M & B & $\checkmark$ & $\checkmark$ & & & & $\checkmark$ & $\checkmark$ & $\checkmark$ & $\checkmark$ & & $\checkmark$ \\
\hline $\mathbf{4 3}$ & Sharp & M & C & $\checkmark$ & $\checkmark$ & & & & $\checkmark$ & $\checkmark$ & & & & $\checkmark$ \\
\hline $\mathbf{4 4}$ & Cyclist & M & B & $\checkmark$ & $\checkmark$ & & $\checkmark$ & $\checkmark$ & $\checkmark$ & $\checkmark$ & & & $\checkmark$ & \\
\hline $\mathbf{4 5}$ & Sharp & M & B & $\checkmark$ & $\checkmark$ & & $\checkmark$ & $\checkmark$ & $\checkmark$ & $\checkmark$ & & & & $\checkmark$ \\
\hline $\mathbf{4 6}$ & Blunt & M & B & $\checkmark$ & $\checkmark$ & & & & $\checkmark$ & $\checkmark$ & & $\checkmark$ & & $\checkmark$ \\
\hline $\mathbf{4 7}$ & Blunt & M & B & $\checkmark$ & $\checkmark$ & & & & $\checkmark$ & $\checkmark$ & & & $\checkmark$ & \\
\hline $\mathbf{4 8}$ & Sharp & M & B & $\checkmark$ & $\checkmark$ & $\checkmark$ & & & $\checkmark$ & $\checkmark$ & & & & $\checkmark$ \\
\hline $\mathbf{4 9}$ & Blunt & M & B & $\checkmark$ & & & & & $\checkmark$ & $\checkmark$ & & $\checkmark$ & & \\
\hline $\mathbf{5 0}$ & PVA & M & B & $\checkmark$ & $\checkmark$ & & $\checkmark$ & $\checkmark$ & $\checkmark$ & $\checkmark$ & & & & $\checkmark$ \\
\hline $\mathbf{5 1}$ & Manual & F & B & $\checkmark$ & $\checkmark$ & & & & $\checkmark$ & & & & $\checkmark$ & \\
\hline $\mathbf{5 2}$ & PVA & M & W & $\checkmark$ & $\checkmark$ & $\checkmark$ & $\checkmark$ & $\checkmark$ & $\checkmark$ & $\checkmark$ & & & $\checkmark$ & $\checkmark$ \\
\hline $\mathbf{5 3}$ & Ligature & F & B & $\checkmark$ & & $\checkmark$ & & $\checkmark$ & $\checkmark$ & $\checkmark$ & & & & $\checkmark$ \\
\hline $\mathbf{5 4}$ & Blunt & M & B & $\checkmark$ & $\checkmark$ & & & & $\checkmark$ & $\checkmark$ & & & & $\checkmark$ \\
\hline $\mathbf{5 5}$ & PVA & F & B & $\checkmark$ & $\checkmark$ & & $\checkmark$ & $\checkmark$ & $\checkmark$ & $\checkmark$ & & & & \\
\hline
\end{tabular}

Key:

$\begin{array}{llll}\text { PVA } & \text { Pedestrian Vehicle Accident } & \text { M } & \text { Male } \\ \text { Blunt } & \text { Blunt force assault } & \text { F } & \text { Female } \\ \text { Sharp } & \text { Sharp force assault } & & \\ \text { Multiple } & \text { Multiple modalities } & \text { B } & \text { Black } \\ \text { Manual } & \text { Manual strangulation } & \text { W } & \text { White } \\ \text { Ligature } & \text { Ligature strangulation } & \text { C } & \text { Coloured }\end{array}$

\section{Evidence Detection}

The maximum and mean number of evidence types detected by each tool is represented in Figure 1. The minimum evidence categories detected was 0 and up to a maximum of 10 different evidence types. 


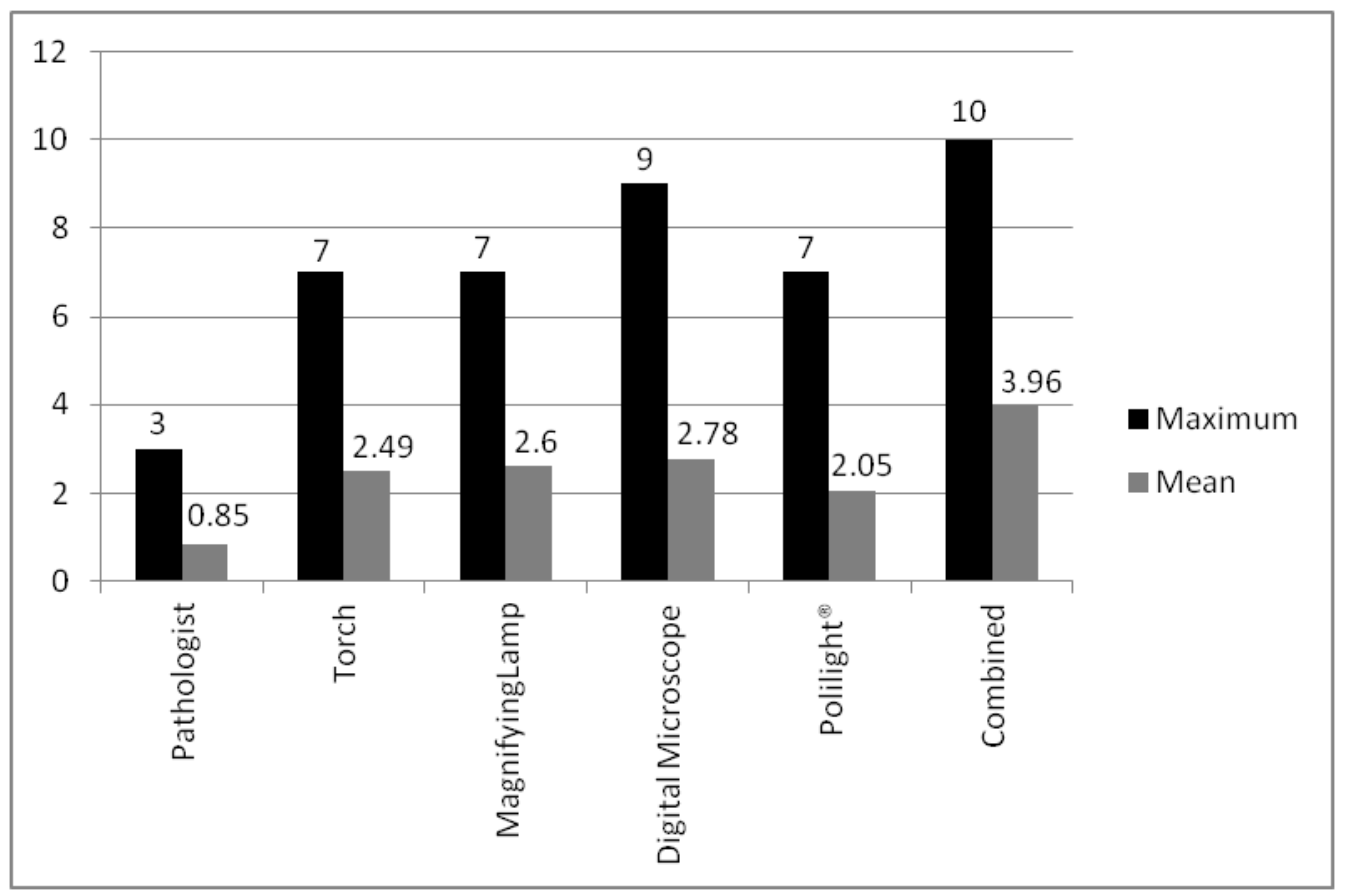

Figure 1. Maximum and mean number of evidence types detected by each technology.

There was no significant difference in the detection of entomological samples, paint, impression marks, fingerprints or other evidence found by the different technologies (McNemar test, $p>0.05$ ). Figure 2 depicts the propensity of each technology for detection of the most common evidence categories.

Table II ranks the technologies in terms of detection ability per evidence category.

\section{Types of samples}

Fibres/hairs were found on 41 out of 55 bodies (74.5\%). Of the 4 cases where a victim was wrapped in a blanket, fibres/hairs were detected on all of them (100.0\%) ( $p>0.05)$. This was not statistically significant, however. Fluid was found on 36 out of 55 bodies (65.5\%). Of the 7 cases where rape or sexual assault was suspected, fluids were found in 5 cases $(p>0.05)(71.4 \%)$. This was not statistically significant. Geological samples were found on 34 out of 55 bodies (61.8\%). Botanical samples were found on 32 out of 55 bodies $(58.2 \%)$. Other evidence was found on 24 of the 55 bodies (43.6\%). Paint smears or chips were found on 13 out of 55 bodies (23.6\%). Of the 8 PVA cases, paint was found on 4 (50.0\%). Glass was found on 9 out of 55 bodies (16.4\%). Of the 8 PVA cases, glass was found in $5(62.5 \%)$ cases. The magnifying lamp was able to detect all of the cases where glass was present. Impression marks were found on 4 out of the 55 bodies (7.3\%). Entomological samples were found on 3 out of the 55 bodies (5.5\%). Fingerprints were found on 1 out of the 55 bodies $(1.8 \%)$. These were found using the torch and the magnifying lamp. The fingerprints were imperceptible using the digital microscope or the Polilight ${ }^{\circledR}$. 


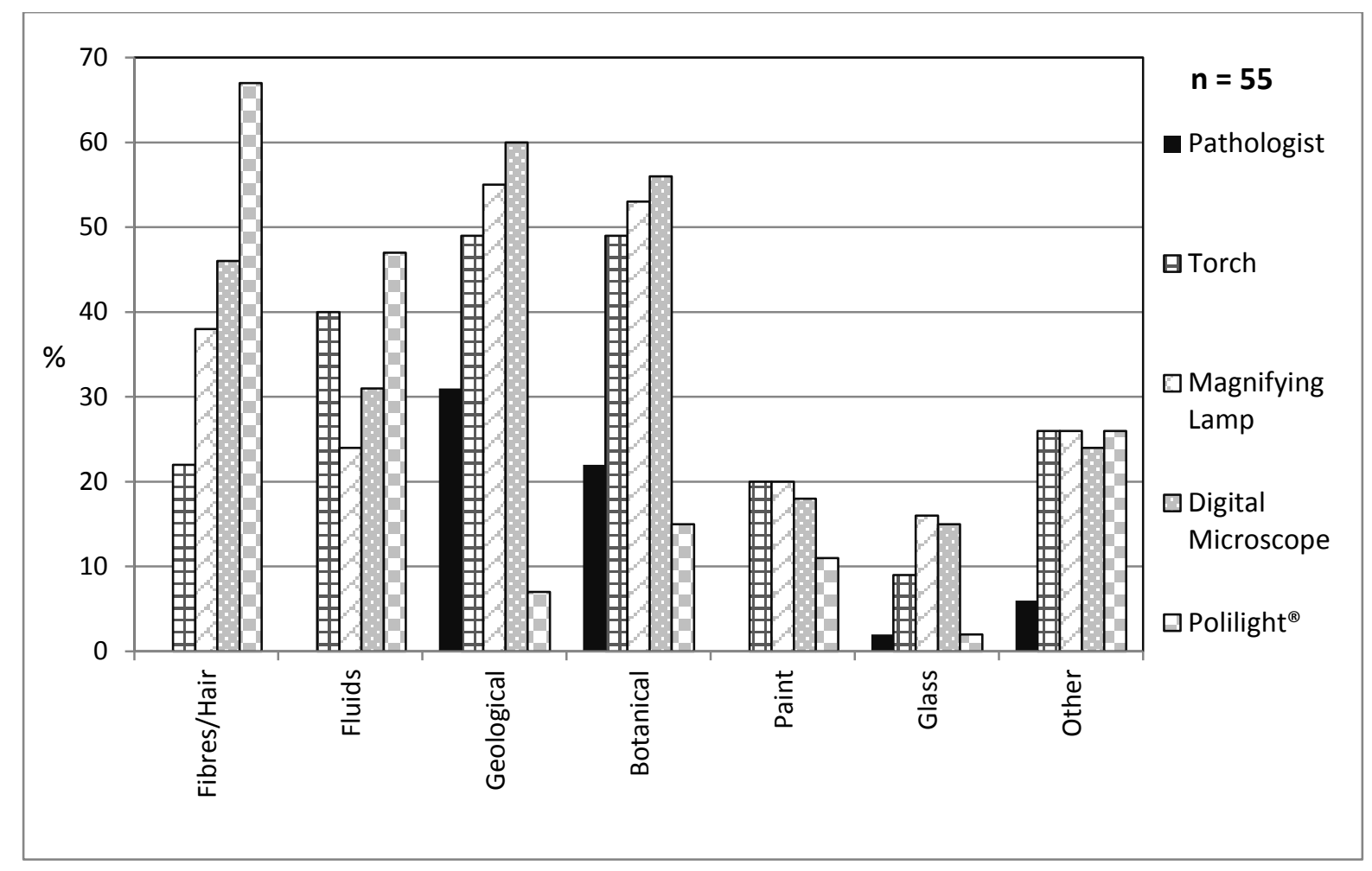

Figure 2. Most common sample types identified using the different technologies.

Table II. Comparison of overall evidence detection ability per evidence type.

\begin{tabular}{|c|c|c|c|c|}
\hline Evidence Type & Torch & Magnifying Lamp & Digital Microscope & Polilight ${ }^{\circledR}$ \\
\hline Botanical & $3^{\text {rd }}$ & $2^{\text {nd }}$ & $1^{\text {st }}$ & $4^{\text {th }}$ \\
\hline Geological & $3^{\text {rd }}$ & $2^{\text {nd }}$ & $1^{\mathrm{st}}$ & $4^{\text {th }}$ \\
\hline Glass & $3^{\text {rd }}$ & $1^{\text {st }}$ & $2^{\text {nd }}$ & $4^{\text {th }}$ \\
\hline Entomological & $2^{\text {nd }}$ & $2^{\text {nd }}$ & $1^{\text {st }}$ & $3^{\text {rd }}$ \\
\hline Plastic & $2^{\text {nd }}$ & $2^{\text {nd }}$ & $1^{\mathrm{st}}$ & $3^{\text {rd }}$ \\
\hline Fingerprints & $1^{\mathrm{st}}$ & $1^{\text {st }}$ & - & - \\
\hline Impression Marks & $1^{\text {st }}$ & $2^{\text {nd }}$ & - & $2^{\text {nd }}$ \\
\hline Tattoos & $1^{\mathrm{st}}$ & $2^{\text {nd }}$ & $3^{\text {rd }}$ & $3^{\text {rd }}$ \\
\hline Faeces & $1^{\text {st }}$ & $1^{\text {st }}$ & $1^{\text {st }}$ & $1^{\text {st }}$ \\
\hline "Red Streaks" & - & - & - & $1^{\text {st }}$ \\
\hline Paper & - & - & - & $1^{\text {st }}$ \\
\hline Fibres/hair & $4^{\text {th }}$ & $3^{\text {rd }}$ & $2^{\text {nd }}$ & $1^{\text {st }}$ \\
\hline Paint & $1^{\text {st }}$ & $1^{\mathrm{st}}$ & $2^{\text {nd }}$ & $3^{\text {rd }}$ \\
\hline Fluid & $2^{\text {nd }}$ & $4^{\text {th }}$ & $3^{\text {rd }}$ & $1^{\mathrm{st}}$ \\
\hline Other Evidence & $1^{\text {st }}$ & $1^{\text {st }}$ & $2^{\text {nd }}$ & $1^{\text {st }}$ \\
\hline
\end{tabular}

Key: - Did not detect 
Red paint chips were found on many of the bodies (See Figure 3), but this was later discovered to most likely originate from the body bags in which the bodies were contained.

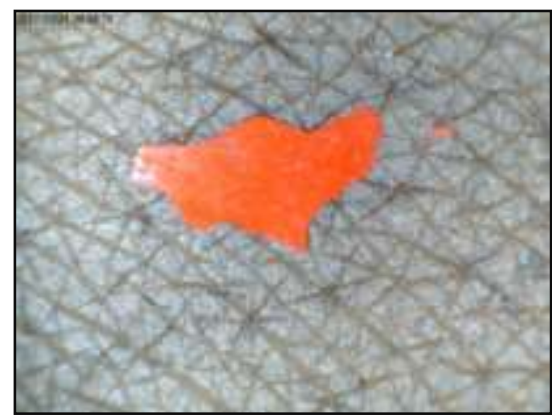

Figure 3. Red paint chips likely originating from the body bags.

"Red Streaks" Indistinct areas of redness seen only by means of the Polilight@ ${ }^{\circledR}$ were noted on some of the bodies (See Figure 4). There was no strict pattern as to the appearance (besides the apparent red colour) or location of these marks. The torch, magnifying lamp and digital microscope did not uncover anything corresponding to the areas where these streaks appeared and none of the pathologists mentioned anything that could be linked to this phenomenon.

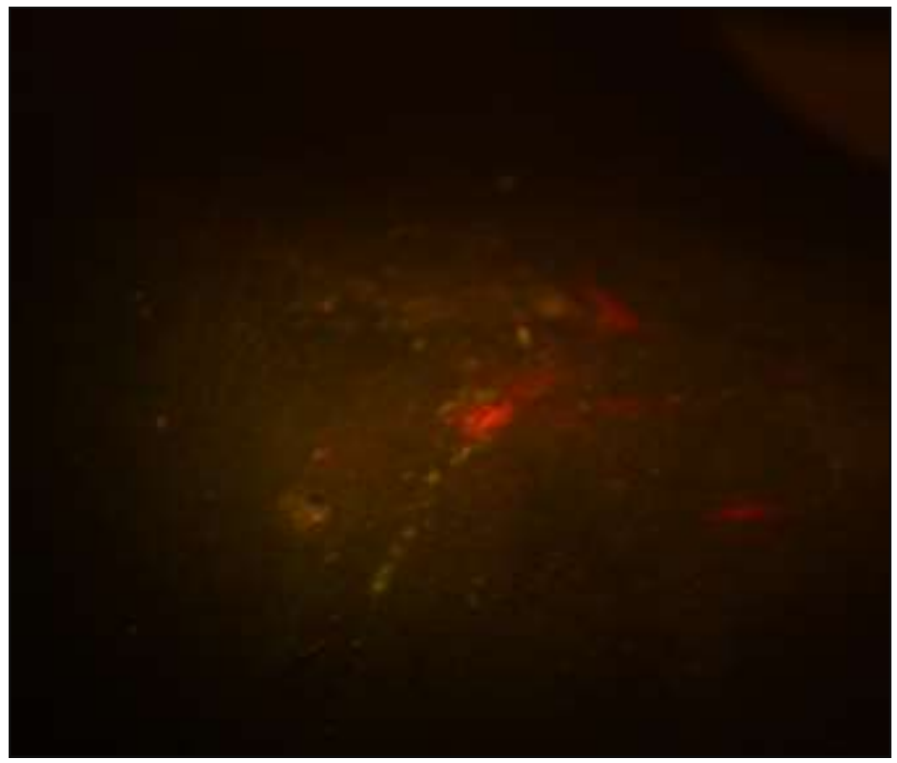

Figure 4. Example of the anomaly "red streaks" seen only under illumination with the Polilight ${ }^{\circledR}$.

Overall Performance

According to the Friedman Test,[7] in terms of quantity of the spectrum of evidence detected, the digital microscope performed best overall followed by the magnifying lamp, torch and the Polilight ${ }^{\circledR}$. This ranking of performance was found to be statistically significant $(p=0.04)$. 
There was a statistically significant difference in the detection of many of the evidence types between the pathologists and the technologies. This applied to botanical samples, geological samples, glass and other evidence $(p=0.00, p=0.00, p=0.021$ and $p=0.00$ respectively).

Evidence categories which did not show a significant difference between the pathologists' detection and that of the tools were impression marks and entomological samples $(p>0.05)$.

\section{Technology Evaluations}

The evaluation of the technologies is summarized in Table III. The torch highlights reflective materials not noticed by other methods and is best suited to detecting impressions, tattoos, paint, and other easily visible evidence.

The magnifying lamp needs to be clamped onto a stationary surface. This rendered the magnifying lamp somewhat impractical for a general body search as manoeuvrability was limited. The magnifying lamp detected glass, paint and other evidence the best.

The digital microscope proved relatively simple and easy to use and is very easy to manoeuvre around the entire body. One drawback is that the device needs to be pressed directly against the surface of interest in order to obtain a clear focussed image which results in soiling the device. The potential for dislodging or contaminating trace evidence that this could cause should be considered. The digital microscope was best at detecting particulate evidence, such as botanical, geological and entomological samples.

The Polilight $\AA$ highlights evidence which would not be seen with the other technologies under normal lighting. It is comparatively very expensive and the model used is very heavy, large and needs to be plugged into a mains power supply. The Polilight $₫$ 's strength lay in fluid and fibre/hair detection.

\begin{tabular}{|l|l|l|l|l|}
\hline & Torch & Mag. Light & Dig. Micro. & Polilight ${ }^{\circledR}$ \\
\hline Price & R500.00- & R400.00- & R300.00- & R38 000.00- \\
& R800.00 & R1000.00 & R2000.00 & R450 000.00 \\
\hline Weight & Light & Medium & Light & Heavy \\
\hline Ease of use & Easy & Medium & Medium & Medium \\
\hline Portability & High & Low & Medium & Low \\
\hline Time & Low & Medium & High & Low \\
\hline Training Required & No & No & Some & Yes \\
\hline Evidence Detection Ability & 3rd & 2nd & 1st & 4th \\
\hline
\end{tabular}

Table III. Summary of technology evaluation

\section{Limitations}

Multiple parameters were being assessed concurrently. The researcher had no control over how the body was handled or transported and trace evidence could have been dislodged during the admission process to the mortuary and subsequent management of the bodies. The death scenes were not attended by the researcher and the presence or absence of 
trace evidence at the scene compared to the examination in the mortuary could not be studied.

\section{Discussion}

A victim of crime's body should be treated with the same care, diligence and vigilance as the physical crime scene; and trace evidence should be meticulously sought for in the same manner. South Africa has earned notoriety as one of the crime capitals of the world,[3] with 4 major cities ranked in a study listing the 50 Most Violent Cities in the World.[8] The violence means a large case load admitted for medico-legal investigation. Despite the increasing crime rate in South Africa, conviction rates have decreased over the years; with the most severe crimes of murder and rape having the lowest conviction rates.[9] Prosecution rates are also on the decrease, with prosecutors choosing to pursue only cases which have a high likelihood of conviction.[10] Approximately only $11 \%$ of all reported crimes are prosecuted in South Africa.[10] This is partly because cases without sufficient evidence to prove guilt beyond a reasonable doubt are abandoned.[10] The reality is that this country's approach to crime solving is not evolving at the same rate that crimes are being committed. One reason for this stagnation may be that, as a developing country, South Africa's resources are limited.

There is virtually no limit to the traces that could be found on a victim's body, albeit each with varying degrees of uniqueness and subsequent usefulness to the investigation. This evidence can prove that a suspect came in contact with the victim around the time of the crime, and can subsequently serve to incriminate or exonerate the individual.[2] In the current study conducted at the PMLL, cases of interpersonal violence were studied with additional technologies in an attempt to improve trace evidence detection. Fifty-five cases were identified and included in the study. Sharp force trauma was the most common cause of death for victims in our study which may have resulted in fewer traces being present for detection, as a weapon was more commonly between the victim and the assailant; whereas more physical-contact orientated causes of death - such as manual and ligature strangulation - would have potentially yielded more proof of contact between the victim and perpetrator. Only 7 cases of suspected rape were included and this could be the reason why the Polilight $\AA^{\circledR}$ 's renowned ability for body fluid detection was not experienced in the current study.

The racial distribution was in keeping with the mortuary population at the PMLL. The colour, absorbency and inherent fluorescence capabilities of the substrate on which a sample is deposited all influence the visibility and enhancement of the deposited sample.[11] It was hypothesized that evidence detection by the Alternative Light Source would be diminished on darker-skinned victims. Due to the fact that the number of Black victims greatly outnumbered the number of White victims in this study, the hypothesis was not proved. Race was found to not be significantly associated with the increased or decreased detectability of any particular evidence types, or to all evidence in general. It is therefore suggested that that the results of international studies[12-13] conducted on fair-skinned individuals are applicable to South Africa's predominately Black population.

Trace evidence offers two fundamental issues in its detection: firstly, the evidence is often minute and present in such a finite amount that the likelihood of noticing it with the naked eye is very low; and secondly, the evidence (e.g. fluids or latent fingerprints) often has a low 
contrast to the background it is deposited on, making its detection highly improbable to nearimpossible.[14] In the study conducted at the PMLL, a torch, magnifying lamp, digital microscope and Polilight $\AA$ were used to search for trace evidence.

The torch ranked third in usefulness at detecting trace evidence in the current study. It is a small and simple light source. It highlights reflective materials not noticed by other methods, but most of the evidence detected with the use of the torch was already visible with the naked eye. This suggests that a well-lit examination environment can mostly replace the need for a torch.

The magnifying lamp ranked second in the PMLL study and proved slightly impractical for a general body search. A portable handheld device would be easier to use and more efficient. The small field of view means that only one person can look at a time, making consultation with colleagues difficult. Also, one needs the lens to be close to the object being inspected as well as one's face, increasing the risk of dislodging evidence or contaminating the subject as well as occupational disease hazards to the investigator. The effectiveness of detecting glass particles and paint in an environment where numerous fatalities due to pedestrians in road traffic accidents are admitted justifies the relatively cost-effective tool.

The digital microscope proved relatively simple and easy to use and was the best in detecting trace evidence in the current study. It is lightweight and compact, images and videos can be captured and considering its capabilities, it is very cost effective. Due to the fact that the microscope must be pressed against the area to be examined, it is probably better suited to examining specific particles rather than locating them. The digital microscope detected geological and entomological samples the best and should be most useful in cases where evidence such as foreign flora may indicate foul play.

The Alternative Light Source used in the study at the PMLL was the Polilight ${ }^{\circledR}$ and it performed the worst in trace evidence detection. An Alternative Light Source is a high intensity light source using a bulb which emits light in the visible, UV (ultra-violet) and IR (infra-red) spectrums.[15] These are in common use in laboratories (both local and international) to guide the examination of clothing and other exhibits.[11] The Polilight巴 ${ }^{\circledR}$ is potentially oversensitive for non-contributory specimens to the point of being distracting from more significant findings. For example, the Polilight ${ }^{\circledR}$ may uncover innumerable fibres on a victim's body, but this has little evidential value if they originate from the blanket in which the body was wrapped and transported to the mortuary. By using an Alternative Light Source exhibits can be searched for evidence without having to handle the surface in question, thus reducing the chance of dislodging traces, smearing prints or contaminating the sample.[15] The best general wavelength and goggle combination for detecting biological stains is 450nm with orange goggles.[16-17] It does not pose any known harmful effects to the user except when operated in the UV range[18] or when the high intensity beam is looked upon without the provided protective eyewear[16] and it is deemed a safe, simple, non-invasive and non-destructive tool for the screening of scenes and exhibits.[19]

Most studies using Alternative Light Source systems focus on inert substrates which limits its applicability to the examination of human skin.[12] In other studies, the substances were allowed to dry before examination.[12,20-21] In the study at the PMLL, the cases were examined after refrigeration which caused many of the biological samples deposited on the bodies to be in liquid state either from the scene or due to condensation created by the 
refrigerator. This may explain why readily visible wet samples were better seen by means of reflection than fluorescence in this study.

Although searching the scene and victim's body for evidence is part of police work, it is suggested that the relatively controlled and less pressured environment of the mortuary may be more conducive to the detailed examination for trace evidence, and may result in more traces being found than at the crime scene. In the very least, these ancillary investigations can corroborate and confirm scene findings. The additional time investment would need to be considered by the facility manager. It is suggested that these examinations be conducted by individuals other than the pathologists so as to reduce the additional time required and minimize disruption to the autopsy.

The nature of the deaths of the majority of subjects studied here necessarily meant that both the bodies and clothing of many of the victims were covered in blood. Blood, vaginal deposits and faeces can mask the fluorescence of semen on fabric.[11] It should be realised that other possible evidence may have been obscured by more superficially deposited substances and this factor may have further reduced the amount of evidence detected in this study.

Portable equipment eliminates the difficulties related to manoeuvring the body of a victim under investigation.[22] The evidence in question is left untouched and can undergo sampling, testing and analysis as if the screening had never happened.

Despite the digital microscope performing statistically the best in this study and the Polilight ${ }^{\circledR}$ performing the worst, it must be kept in mind that each tool is best suited for different evidence types and this consideration is seen to bear more weight than generalized statistics. It is the researcher's recommendation that for an environment such as the PMLL, investment in a unit such as the Polilight ${ }^{\circledR}$ as well as the magnifying lamp would aid the investigation of most of the cases admitted. Rape-homicides are the biggest application for the Polilight ${ }^{\circledR}$ at the PMLL and additional time expenditure is justified for these cases. Each institution needs to review their current practice in order to decide which of these modalities - if any - would add value to their establishment.

\section{Conclusion}

It was found that these tools do have applicability in the medico-legal mortuary setting. The Polilight ${ }^{\circledR}$ is relatively expensive and best suited to detecting biological traces. The torch is lightweight and best for locating reflective particles and fluids. The magnifying lamp is useful for locating small particles that, although visible with the naked eye, are more easily detected at a slightly higher magnification; but a portable version would be more desirable. The digital microscope is cost-effective and best suited to examining specific particles or lesions, rather than locating them initially. More rigorous studies would need to be done in order for a mortuary manager to be able to make informed decisions regarding the expenditure of additional funds, time, training and effort at their specific institution.

\section{References}

[1] Locard E. Analyses of dust traces parts I, II and III. Am J Police Sci 1930;1:276-298, 401-418, 496-514.

[2] Virkler K, Lednev IK. Analysis of body fluids for forensic purposes: From laboratory testing to nondestructive rapid confirmatory identification at a crime scene. Forensic Sci Int 2009;188:1-17. 
[3] Reza A, Mercy JA, Krug E. Epidemiology of violent deaths in the world. Injury Prevent 2001;7:104-111.

[4] United Nations. Over 270,000 pedestrians killed on roads every year. UN agency. UN Publications. United Nations, New York. 2 May 2013. See http://www.un.org/apps/news/story.asp? News|D=44815\&Cr=road+ safety\&Cr1 (Accessed 3 October 2013).

[5] Burton JL. The external examination: An often-neglected autopsy component. Mini-symposium: The autopsy in sudden unexpected adult death. Curr Diag Path 2007 Oct;13(5):357-365.

[6] Donson H (ed.). A Profile of Fatal Injuries in South Africa. Ninth Annual Report of the National Injury Mortality Surveillance System. MRC-UNISA Crime, Violence and Injury Lead Programme, 2007.

[7] Friedman M. The use of ranks to avoid the assumption of normality implicit in the analysis of variance. $J A m$ Stat Assoc (American Statistical Association) 1937 Dec; 32(200):675-701.

[8] San Pedro Sula, la ciudad mas violenta del mundo, jaurez, la segunda. Seguridad, Justicia y Paz January 2012. See http://www.seguridadjusticiaypaz.org.mx (Accessed 2 February 2012)

[9] Mistry D. Victims and the Criminal Justice System in South Africa. Centre for the Study of Violence and Reconciliation, Johannesburg, Oct 1997.

[10] Schönteich M. Assessing the crime fighters: The ability of the criminal justice system to solve and prosecute crime. Occasional Paper No.40. Institute for Security Studies, Sept 1999.

[11] Kobus HJ, Silenieks E, Schamberg J. Improving the effectiveness of fluorescence for the detection of semen stains on fabrics. J Forensic Sci 2002;47:819-823.

[12] Wawryk J, Odell M. Fluorescent identification of biological and other stains on skin by the use of alternative light sources. J Clin Forensic Med 2005;12:296-301.

[13] Hughes VK, Ellis PS, Langlois NEI. Alternative light source (Polilight) illumination with digital image analysis does not assist in determining the age of bruises. Forensic Sci Int 2006;158:104-107.

[14] Ricki L. DNA fingerprints: witness for the prosecution. Discover 1988 Jun.

[15] SPEX Forensics Forensic light source applications: wavelengths and uses. The who, what, where, and when of wavelengths. 1.800.GET.SPEX - 1.732.623.8335. p2-5. See http://www.horiba.com/fileadmin/uploads/Scientific/Documents/Forensics/fls.pdf (Accessed 5 August 2013).

[16] Rofin Polilight PL500 Version 2 Instruction Manual, Australia: Rofin; 2001.

[17] Vandenberg N, van Oorschot RAH. The use of Polilights in the detection of seminal fluid, saliva, and bloodstains and comparison with conventional chemical-based screening tests. J Forensic Sci 2006 Mar;51(2):361-370.

[18] Webb JL, Creamer JI, Quickenden TI. A comparison of the presumptive luminol test for blood with four nonchemiluminescent forensic techniques. Luminescence 2006;21:214-220.

[19] Schulz MM, Wehner F, Wehner HD. The use of a tunable light source (Mini-Crimescope MCS-400, SPEX Forensics) in dissecting microscopic detection of cryptic epithelial particles. J Forensic Sci 2007 Jul;52(4):879-883.

[20] Nelson DG, Santucci KA. An alternate light source to detect semen. Acad Emerg Med 2002 Oct;9:10451048.

[21] Stoilovic M. Detection of semen and blood stains using Polilight as a light source. Forensic Sci Int 1991;51:289-296.

[22] Khursheed A, Nelliyan K, Ding Y. Nanoscale imaging with a portable field emission scanning electron microscope. Microelectron Eng 2006:83;762-766. 